\title{
DEVELOPMENTAL PHARMACOLOGY
}

INTERPRETATION OF PUTATIVELY TOXIC DIGOXIN SERUM 385 CONCENTRATIONS IN CHILDREN, Gideon Koren, Ruth Div, of Clin. Pharmacology, The Hosp. for Sick Children, Toronto. Between January 1981 and April 1984 serum concentrations of digoxin in excess of $5 \mathrm{ng} / \mathrm{ml}$ were recorded in $47 \mathrm{children} \mathrm{(age}$ range 2 days to 16 years). In 10 of them high concentration were measured 9-48 hours after death and were significantly higher than antemortem levels in all cases $(8.3 \pm 2.4$ vs $3.3 \pm$ $1.5, \mathrm{P}<0.0001)$. In 15 cases $(40.5 \%$ of the living patients) serum concentrations of $\geq 5 \mathrm{ng} / \mathrm{ml}$ reflected sampling errors in that drug concentrations were monitored too soon after administration. On subsequent drug discontinuation later serum determinations were not in the toxic range. None of these children exhibited clinical digoxin toxicity. In 9 cases the excessive concentrations could be associated with renal failure and prolonged $\mathrm{T}^{\frac{1}{2}}$ of digoxin. In only two of these children were ther signs of digoxin toxicity. Six cases were caused by digoxin overdose (accidental ingestions, dispensing error, suicide attempt, and excessive dose for age). In 6 additional cases the presence of an endogenous digoxin like substance (EDLS) could be shown as a component of elevated drug concentration. One case could be attributed to digoxin-amiodarone interaction. Overal1, in 10/37 living patients digoxin toxicity was diagnosed. These observations suggest that serum concentrations of digoxin in excess of $5 \mathrm{ng} / \mathrm{ml}$ do not uniformly predict toxicity. Sampling errors, postmortem determinations and circulating EDLS should be considered as possible alternative explanations.

NDOGENOUS DIGOXIN LIKE SUBSTANCE(S) (EDLS) IN NEW-

386 BORN INFANTS. Gideon Koren, Steve Soldin, Stuart M. MacLeod. Div. of Clin. Pharmacology \&

We measured digoxin serum concentrations by RIA in 30 neonates not receiving digoxin (gestational age $33.5 \pm 4.8 \mathrm{wk}$, range 25-40). Values ranged from 0.17 to $1.64 \mathrm{nM}$ (mean $0.64 \pm$ $0.27 \mathrm{nM})$. Gestational age and concentration of EDLS were negatively correlated $(r=-0.62, P<0.01)$. In an additional 6 critically ill newborn babies and infants receiving digoxin in adequate doses, excessive values of digoxin ( $>6 \mathrm{nM}$ ) could be explained in part by circulating EDLS. In 4 of them digoxin explained in part by circulating EDLS. In 4 of them digoxin concentrations continued to rise after cessation of therapy to
concentrations as high as $14 \mathrm{nM}$. In the 2 remaining babies digoxin concentrations fell appropriately but stabilized at values of $1.5-2 \mathrm{nM}$ suggesting that this concentration represents EDLS. Cross-reactivity studies comparing RIA and fluorescent polarization immunoassay show that coxtisone is the most immunoreactive substance in cord serum. EDLS was successfully extracted and concentrated from cord serum and placentas and was separated into several immunoreactive fractions by reverse phase HPLC. A potent inhibitor of $\mathrm{Nat} / \mathrm{Kt}$ - ATPase has been identified HPLC. A potent inhibitor of Nat/Kt - ATPase has been identified in a progesterone-containing fraction. Simulation pharmaco-
kinetics suggest that the presence of $0.6 \mathrm{nM}$ EDLS would lengthen digoxin $\mathrm{T} \frac{1}{2}$ by $64 \%$, reduce Vd by $23 \%$ and lessen clearance by $53 \%$ if "true" peak digoxin level was $2 \mathrm{nM}$ and "true" $\mathrm{T} \frac{1}{2}=28 \mathrm{hr}$. It appears likely that currently accepted digoxin kinetic parameters for infants are approximations only and distortion of phar macokinetic behaviour by EDLS may lead to inappropriate dosing.

PHARMACOKINETICS OF NETILMICIN IN NEWBORN INFANTS.

387 Robert J. Kuhn, Milap C. Nahata, Rex A. Bickers, 387 Dwight A. Powell, Colleges of Pharmacy and Medicine, Ohio State University, Children's Hospital Department of Pediatrics, Columbus, Ohio.

Netilmicin, a recently released aminoglycoside is used in neonates as an alternative to gentamicin or tobramycin for treatment of suspected or proven sepsis. We studied netilmicin kinetics in 8 premature infants $(4 \mathrm{M}, 4 \mathrm{~F})$ with an average gestation age of 31.3 weeks (range 28-33), a postnatal age of 3 days, and birth weight of 770-1640 grams. Patients were receiving netilmicin, $2.5 \mathrm{mg} / \mathrm{kg} / \mathrm{IV}$ over 0.33 hours every 12 hours for presumed or proven sepsis. Patient who weighed 770 grams was presumed or proven sepsis. Patient on $18^{\circ}$ dosing schedule. At steady-state, blood samplaced. on $\mathrm{q} 18^{\circ}$ dosing schedule. At steady-state, blood samples were obtained at $0,0.5,1,2,4,8$ and 12 hours after starting the infusion and analyzed by $\mathrm{TDx}$, thod. Peak and
trough serum concentrations of netilmicin ranged $6.7-12.6$ (9.7 $2.4) \mathrm{mcg} / \mathrm{ml}$ and $2.2-3.7(2.81 \pm .5) \mathrm{mcg} / \mathrm{ml}$, respedtively. Seven of eight patients had trough serum concentration $>2 \mathrm{mcg} / \mathrm{ml}$. Total body clearance of netilmicin ranged from $0 . \overline{3} 7$ to 0.95 $(.72 \pm .21) \mathrm{ml} / \mathrm{min} / \mathrm{kg}$. Apparent volume of distribution ranged from 0.33 to $0.82(.52 \pm .18) \mathrm{L} / \mathrm{kg}$ and elimination half-life from 4.6 to $11.5(8.3 \pm 2.7)$ hours. Netilmicin clearance appeared from 4.6 to $11.5(8.3 \pm 2.7)$ hours. Netilmicin clearance appeared body weight. A 2- to 3-fold variation in the kinetic parameters and the frequent occurrence of trough serum concentrations $>2 \mathrm{mcg} / \mathrm{ml}$ indicates the need for close monitoring of netilmicin therapy in newborn infants.
388 ANALOGUE AND THEOPHYLLINE IN NON388 ANAESTHETIZED RABBIT PUPS. Hugo Lagercrantz, $M$. Runold, and B. Fredholm. Nobel Institute for Neurophysiology, Karolinska Institute, Department of Pharmacology, and Karolinska Hospital, Department of Pediatrics, Stockholm, Sweden. (Sponsored by Hakan Sundell)

Recent studies have indicated that the respiratory stimulatory effect of theophylline in neonates is rather due to antagonism of endogenously released adenosine than by inhibition of phosphodiesterase. This was tested in non-anaesthetized rabbit pups. $A$ adenosine analogue $\mathrm{L}-\mathrm{N}^{6}$-phenyl-isopropyl-adenosine (PIA) was injected i.p. in 17 pups. Respiratory volumes were recorded with the barometric method during natural sleep. Ventilatory volumes barometric method during natural sleep. Ventilatory volumes decreased $65.2+6.0 \%$ (SD, $n=4) 15$ min. after PIA was given to the one
day old pups, while the effect was less pronounced in the 3 and 8 days old pups $(-43.4 \pm 5.8 \%, n=4 ;-31.0 \pm 13.5 \%, n=3)$. A linear dosedependent respiratory depression was found in the concentration range from $0.01-1.0$ umol $/ \mathrm{kg}$. The respiratory depressive effect could be completely blocked with theophylline pretreatment $(10 \mathrm{mg} / \mathrm{kg})$. We found a higher affinity for PIA in the brains from the youngest pups possibly explaining their higher susceptibility. PIA was also found to decrease body temperature, oxygen consumption and cause sedation of the animal. All these effects were reversed with theophylline. These results indicate that adenosine which is released by hypoxia, might be one factor causing respiratory depression--effects which can be antagonized by theophylline.

CYTOCHROME P-450-P; A PREGNANCY (P) INDUCED

389 P-450 ISOZYME IN MICE. George H. Lambert, Helen Pritzker Sch. of Med., Univ. of Chgo.; Michael Reese Hosp., Depts. Ped, Pharm. and Physiol. Sci., Chicago Ill. Cytochrome P-450 mediated mixed function monooxygenases (P-450) are a group of enzymes which metabolize many substrates including steroids, environmental chemicals and drugs. P-450 in animals has been shown to be important in certain chemical induced birth defects, cancer, mutations and organ damage. Select $\mathrm{P}-450$ isozymes can be induced by exposure to certain chemicals. We and others have shown that $\mathrm{P}$ markedly decreases maternal hepatic P-450 function. To further investigate the effect of $P$ on $P-450$ we used an HPLC method (PNAS
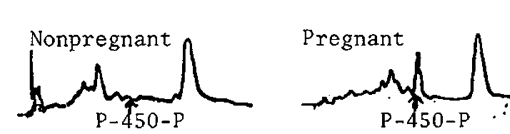
$77: 64731980$ ) to analy pregnant C57BL/6 mice were compared. Comparison of elution profiles of $\mathrm{P}-450$ from nonpregnant and pregnant mice show a marked increase during $P$ in a specific peak (P-450-P) as shown. P-450-P did not co-chromatograph with the classical P-450 isozyme peaks induced by phenobarbital, 3 methylcholanthrene, or pregnenolone-16 $\propto$ carbonitrile. P-450-P is detectable by day 6 of gestation and remains elevated at least through post partum day 3 . In summary, $P$ induces in mice a new major Ppost partum day 3 . In summary, $P$ induces in mice a new major $P-$
450 isozyme peak, P-450-P, which does not co-chromatograph with the classical forms of $\mathrm{P}-450$.

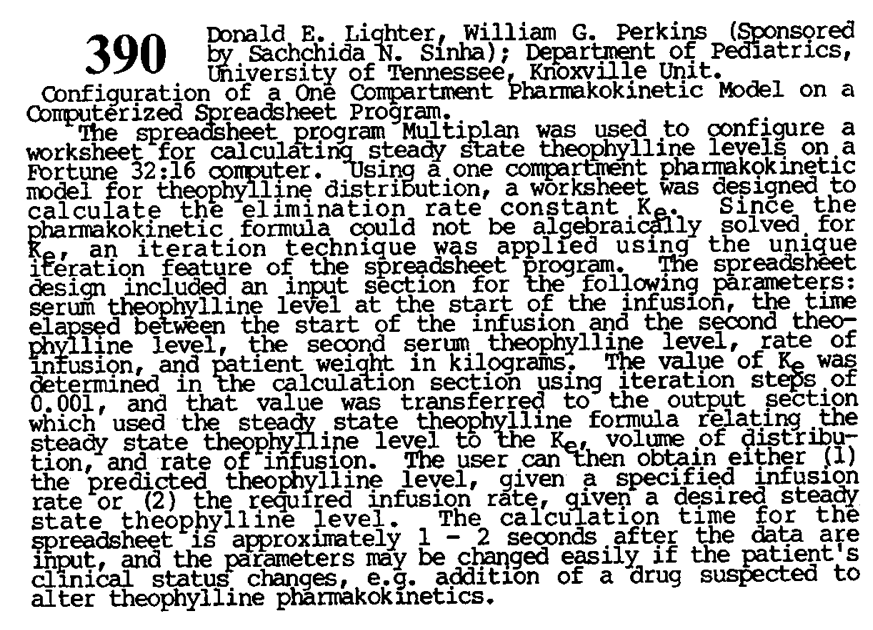

